\title{
Quality in pathology
}

\author{
J. H.J.M. van Krieken ${ }^{1}$
}

Received: 5 July 2017 / Accepted: 7 July 2017 /Published online: 18 July 2017

(C) Springer-Verlag GmbH Deutschland 2017

Many years ago, I read Robert M. Pirsig's "Zen and the Art of Motorcycle Maintenance" for the first time and have read it a couple times again since. It deals with many things, and like all good literature, it has no single meaning or message, but key to it is an investigation into "quality." An important element I took from it is that quality cannot be defined by numbers and is not purely rational. It is, at least partly, an experience, a feeling and key to being human. Virchows Archiv has decided to launch a special section entitled "Quality in Pathology," an idea that I welcome very much. But why is such an extra focus for Virchows Archiv important? What does it encompass?

Pathologists are very proud of their reliability; a cancer diagnosis is almost always correct, and although there are some issues regarding grading and classification, the vast majority of patients are correctly treated, thanks to very effective and cheap pathology. Furthermore, clinicians and patients generally have a great trust in pathology. So, what is the need for spending resources on researching quality?

One common criticism is that quality programs are bureaucratic and cost time without delivering anything. Yet there are several reasons why a focus on quality in pathology is relevant, reasons that are partly coming from society as a whole and partly thanks to the development of our profession. In general, our society is one of a high level of trust, meaning that citizens, and in our case patients, trust professionals and institutions. As we all know, some are trusted more (doctors, engineers) than others (politicians, journalists) but there is a

J. H.J.M. van Krieken

j.vankrieken@pathol.umcn.nl

1 Department of Pathology, Radboud University Medical Center, P.O. Box 9101, 6500 Nijmegen, HB, Netherlands gradual decline in trust. Increasingly, individuals and organizations are requested to show that they are trustworthy, not to simply tell us that they are. Whether we like it or not, we-as pathologists - are part of that society, paid by it, and therefore have to show that what we do is of high quality. This has resulted in the introduction of accreditation programs, often based on ISOnorms. Such programs do not intend to increase quality, but to show it. However, a large problem in pathology is the fact that quality programs focus on the laboratory processes, with little to no focus on the actual diagnosis provided by pathologists.

Pathology itself is changing too, especially living as we are in the era of precision medicine. Pathologists not only provide a diagnosis, but often additional precise information, like on a specific mutation or protein expression, that determines the treatment (and thus outcome!) of the patient. The role of pathology has increased enormously and thus also has its responsibility: pathologists are seen more and more as part of the treatment team. Beneath this lies the notion, that the pathologist and patient need to rely on the outcome of a single test. Where a good pathologist can make a fine diagnosis on a poor H\&E stain and some adequate immunostains (but not the other way around!), no one can make the correct call based on an incorrect DNA test. Many articles in recent years, including several published in Virchows Archiv, have shown how important external quality assessment is; for instance, RAS-testing on colorectal cancer specimens and how we need to improve quality there. Even in 2017, many laboratories still make mistakes in this relatively simple molecular test.

Given the importance of quality demonstration and assessment in pathology, there is need for a forum that enables the scientific debate on the topic. I fear the articles will not be held in the same page-turning regard as Pirsig's Zen..., but they will be of great relevance. We need to better understand what we mean by "quality" in pathology and guidance on how to have efficient quality systems with as little bureaucracy as 
possible. How do we improve the reliability of our testing, the quality of grading, and classification where relevant? What is already good and what needs improvement? I hope that the new section in Virchow's will provide a forum for such articles and also opinion papers. Maybe there could even be a related web forum for discussion on these topics. In fact, I do believe that we can only maintain a high level of trust in pathology when we have high-quality diagnoses and can show this to the patient. Without trust in our profession, we will not survive. A great challenge for the new section!

Han van Krieken 